\title{
Overexpression of rhophilin 2 promotes pancreatic ductal adenocarcinoma
}

\author{
WENTAO BO, XIELIN FENG and XIAOLI TANG \\ Department of Hepatopancreatobiliary Surgery, Sichuan Cancer Hospital \& Institute, Sichuan Cancer Center, School \\ of Medicine, University of Electronic Science and Technology of China, Chengdu, Sichuan 610041, P.R. China
}

Received May 13, 2020; Accepted October 16, 2020

DOI: $10.3892 / \mathrm{ol} .2020 .12337$

\begin{abstract}
Pancreatic ductal adenocarcinoma (PDAC) is the most common type of pancreatic cancer and is the seventh leading cause of global cancer deaths. In recent years, targeted therapy has been used for pancreatic cancer; however, the drugs available for use in targeted therapy for pancreatic cancer are still very limited. Hence, identification of novel targeted molecules for PDAC is required. Rhophilin 2 (RHPN2) was proven to be a driver gene in glioblastoma. However, the function of RHPN2 in PDAC remains unknown. In the present study, the function of RHPN2 was investigated. The RHPN2 levels were overexpressed by pcDNA3.1-RHPN2 and downregulated by si-RHPN2.Cell proliferation was assessed using the MTT assay and apoptosis was assessed using flow cytometry. The results revealed that high RHPN2 levels in PDAC tissue were correlated with a low overall survival rate of patients with PDAC. Inhibition of RHPN2 reduced SW1990 and PANC1 proliferation and increased the rate of apoptosis. Network analysis demonstrated that centrosomal protein 78 expression was negatively correlated with RHPN2 expression. In conclusion, the present study demonstrated that RHPN2 may promote PDAC making it a potential candidate for targeted therapy.
\end{abstract}

\section{Introduction}

Pancreatic cancer is the seventh leading cause of global cancer deaths in industrialized countries (1) and the third most common in the USA (2). Chinese people increasingly live in urban areas; when combined with other factors, such as aging and environmental pollution, this has shifted the disease spectrum in China from infectious to non-infectious diseases and the health burden of cancer is increasing (3). In 2010, 34,509 men

Correspondence to: Dr Xiaoli Tang, Department of Hepatopancreatobiliary Surgery, Sichuan Cancer Hospital \& Institute, Sichuan Cancer Center, School of Medicine, University of Electronic Science and Technology of China, 55 People South Road Section 4, Chengdu, Sichuan 610041, P.R. China

E-mail: xiaolitangsc@163.com

Key words: cellular proliferation, cell apoptosis, rhophilin 2, pancreatic ductal adenocarcinoma and 23,226 women died from pancreatic cancer in China, with the number of deaths exceeding that in the United States $(4,5)$. Pancreatic ductal adenocarcinoma (PDAC) is a type of exocrine pancreatic cancer and is the most common type of pancreatic cancer with 95\% of all pancreatic cancer cases being PDAC (6).

Existing therapies for PDAC leave much to be desired. Surgery is the most commonly used treatment for pancreatic cancer; even so, only $20 \%$ of patients with pancreatic cancer are candidates for surgery as most pancreatic cancers are first diagnosed after the disease has metastasized (7). In recent years, targeted therapy has been employed in pancreatic cancer; for instance, erlotinib is approved by the Food and Drug Administration authority for patients with advanced pancreatic cancer in combination with gemcitabine (8), and larotrectinib (Vitrakvi ${ }^{\circledR}$ ) is approved as a treatment for pancreatic cancer that is metastatic or cannot be removed with surgery (9). However, targeted therapy options for pancreatic cancer remain very limited. Hence, the identification of novel target molecules for PDAC is vitally important.

A previous study of aggressive glioblastoma identified rhophilin 2 (RHPN2) as a novel driver gene of mesenchymal transformation (10). The aforementioned study demonstrated that RHPN2 gene amplification was associated with a dramatic decrease in the overall survival of patients with glioma. RHPN2 has been described as a Ras homolog family member A (RhoA)-binding protein, but its biological function remains unclear $(11,12)$.

The function of RHPN2 in PDAC remains unknown. The aim of the present study was to elucidate the function of RHPN2 in PDAC and identify the possible therapeutic target gene for PDAC therapy.

\section{Materials and methods}

Bioinformatics analysis. cBio Cancer Genomics Portal (https://cbioportal.org) was used to explore the role of RHPN2 in cancer genomics $(13,14)$. The frequency of alterations of RHPN2 and patients' survival data in pan-cancer and pancreatic cancer were exported from the cBio Cancer Genomics Portal. Gene Expression Omnibus (GEO) dataset values were downloaded from the GDS4336/8035980 datasets (45 pairs of PDAC tumor and adjacent normal tissue, Homo sapiens) (15). The protein network of RHPN2 was analyzed using Cytoscape software version 3.8.0 (16). 
Tissue samples. PDAC tissues and matching adjacent normal tissues (61 pairs) were acquired from the Sichuan Provincial Cancer Hospital (Chengdu, China). Patients were recruited from April 2013 to November 2015. The inclusion criteria were as follows: i) No history of any other active cancer; ii) no active cancer treatment; and iii) no past history of pancreatic cancer. The tissue sample were acquired by resection. The distance between the PDAC tissues and matching adjacent normal tissues was $\sim 1 \mathrm{~cm}$. The mean age of the patients was 65.8 years, age range, 44-81 years and there were 27 males and 34 females. Tissue were stored at $-80^{\circ} \mathrm{C}$ before fixation. For long-term preservation, tissues were embedded in paraffin. Patients were followed-up for at least 5 years. The Ethics Committee of Sichuan University (Chengdu, China) approved the use of the human samples (approval no. 20191109). All specimens were used properly in accordance with the protocol of the Ethics Committee. Written informed consent was obtained from all the patients enrolled in the present study. The classification systems for stage and grade used in the present study were from AJCC Cancer Staging Manual 8th edition (17).

Immunohistochemistry (IHC). RHPN2 protein levels were assessed by IHC. Tumor tissue were immersed in $10 \%$ neutral buffered formalin (NBF) for fixation for at least 3 days at room temperature. Then the whole tissues were dehydrated in a gradients of ethanol (incubated $50 \%$ ethanol for $10 \mathrm{~min}, 70 \%$ ethanol for $10 \mathrm{~min}, 80 \%$ ethanol for $10 \mathrm{~min}, 95 \%$ ethanol for $10 \mathrm{~min}$, $100 \%$ ethanol for 10 min thrice). The tissues were exchanged ethanol with xylene in room temperature in the following sequence: 2:1 ethanol:xylene for 10-15 min, 1:1 ethanol:xylene for 10-15 min, 1:2 ethanol:xylene for 10-15 min, 100\% xylene for 10-15 min thrice. Then xylene was exchanged with paraffin in $58^{\circ} \mathrm{C}$ in the following sequence: $2: 1$ xylene:paraffin for $30 \mathrm{~min}$, 1:1 xylene: paraffin for $30 \mathrm{~min}, 1: 2$ xylene:paraffin for $30 \mathrm{~min}$, $100 \%$ paraffin for $2 \mathrm{~h}$ and $100 \%$ paraffin overnight, and then embedded in a fresh new paraffin. Next, $4-\mu \mathrm{m}$ thick paraffin sections were cut. After deparaffinization and hydration (the reverse sequence of paraffinization and dehydration), the slides were microwaved for antigen retrieval. The slides were blocked in BSA blocking buffer (cat. no. 37520; Thermo Fisher Scientific Inc.) at room temperature for $1 \mathrm{~h}$. The slides were then incubated at $4^{\circ} \mathrm{C}$ overnight with anti-RHPN2 (1:1,000; cat. no. PA5-62469; Thermo Fisher Scientific Inc.). After washing three times with phosphate-buffered saline (PBS), the slides were incubated with goat anti-rabbit poly-horseradish peroxidase-conjugated secondary antibody (1:500; cat. no. 32260; Thermo Fisher Scientific Inc.) at room temperature for $2 \mathrm{~h}$. The slides were then developed using diaminobenzidine (DAB) staining (18). The hematoxylin staining was performed using the $H \& E$ stain kit (cat no. ab245880; Abcam). The slide were incubated in hematoxylin, Mayer's (Lillie's Modification) for $5 \mathrm{~min}$ at $4^{\circ} \mathrm{C}$. Then slides were rinsed with two changes of distilled water to remove excess stain. Subsequently, the slides were incubated with bluing reagent from the H\&E stain kit for $10 \mathrm{sec}$. These slides were observed by light microscope Leica DM3000 (Leica Microsystems Ltd.) (magnification, x200). Images were assessed using the Aperio ImageScope software version 12.4.0.5043 (Leica Microsystems).

Cell culture. Pancreatic cancer cell lines PANC1 (cat. no. CRL-1469 ${ }^{\mathrm{TM}}$ ) and SW1990 (cat. no. CRL-2172 ${ }^{\mathrm{TM}}$ ) were purchased from ATCC. PANC1 cells were cultured in DMEM medium (cat. no. 11965092; Thermo Fisher Scientific Inc.) with 10\% fetal bovine serum (cat. no. 12483020; Thermo Fisher Scientific Inc.). SW1990 cells were cultured in Leibovitz's L-15 Medium (cat. no. 11415056; Thermo Fisher Scientific Inc.) with $10 \%$ fetal bovine serum (cat. no. 12483020; Thermo Fisher Scientific Inc.). Cells and medium were cultured at $37^{\circ} \mathrm{C}$ with $5 \% \mathrm{CO}_{2}$. The exponentially growing cells were used for subsequent experiments.

Detection of RHPN2 and CEP78 by reverse-transcription quantitative $(R T-q) P C R$. Total RNA from tumor tissues and PANC1 and SW1990 cells was extracted with TRIzol reagent (Invitrogen; Thermo Fisher Scientific Inc.) following the manufacturer's instructions. Glyceraldehyde-3-phosphate dehydrogenase (GAPDH) was used as an internal control. cDNA synthesis were performed by QuantiTect Reverse Transcription Kit (cat. no. 205311; Qiagen GmbH). Reactions were incubated at $42^{\circ} \mathrm{C}$ for $50 \mathrm{~min}$ followed by heat inactivation for $5 \mathrm{~min}$ at $80^{\circ} \mathrm{C}$ for reverse transcription. The gene expression levels were assessed via qPCR using the $2^{-\Delta \Delta C q}$ method (19). The PCR amplification was performed using SYBR ${ }^{\mathrm{TM}}-$ Green PCR Master Mix (cat. no. 4334973; Thermo Fisher Scientific, Inc.). The primers used were as follows: RHPN2, forward, 5'-AAGGGCTGTAAT CCCCTTGC-3' and reverse, 5'-CCGCACCTTTGAGTTTG TGG-3'; centrosomal protein 78 (CEP78), forward, 5'-TGGCAG GGAGCAGATCACA-3' and reverse, 5'-AAGCCAGCCATA CAGTCAAGA-3'; and GAPDH, forward, 5'-CTGACTTCAA CAGCGACACC-3' and reverse, 5'-TAGCCAAATTCGTTGTC ATACC-3'. Thermocycling conditions consisted of $50^{\circ} \mathrm{C}$ for $2 \mathrm{~min}$ and $95^{\circ} \mathrm{C}$ for $10 \mathrm{~min}$, followed by 40 cycles of $95^{\circ} \mathrm{C}$ for $15 \mathrm{sec}$ and $60^{\circ} \mathrm{C}$ for $60 \mathrm{sec}$. RHPN2 mRNA expression levels in the control were arbitrarily defined as $100 \%$. The relative RHPN2 levels [ $\log _{2}$ (Tumor/Normal)] were calculated.

Transfection. Small interfering (si) RNA-RHPN2 and pcDNA3.1-RHPN2 transfection were performed.siRNA-RHPN2 and pcDNA3.1-RHPN2 were designed and constructed by Shanghai Shengong Biology Engineering Technology Service., Ltd. Scrambled siRNA (si-NC) and pcDNA3.1 were used as controls. The sequences used were as follows: RHPN2, 5'-AAGCTGCGGAGCATTGAGGTG-3' and scrambled siRNA, 5'-GGTGCCGAATTGAGGTGACGA-3'. Cells were seeded into 24 -well plates at a density of $5 \times 10^{4}$ cells/well overnight. Transfection was performed using Lipofectamine ${ }^{\circledR} 2000$ (cat. no. 11668027; Thermo Fisher Scientific, Inc.) according to the manufacturer's instructions. SiRHPN2 $(0.6 \mu \mathrm{g})$ or pcDNA3.1-RHPN2 $(1 \mu \mathrm{g})$ were used separately. Si-NC $(0.6 \mu \mathrm{g})$ or empty plasmid $(1 \mu \mathrm{g})$ were used as control. The cells were transfected at $37^{\circ} \mathrm{C}$ in a $\mathrm{CO}_{2}$ incubator for $24 \mathrm{~h}$. RHPN2 mRNA expression levels of the si-NC transfection group and those of the pcDNA3.1 transfection group were arbitrarily defined as $100 \%$. Cell proliferation analysis was performed at 0, 24, 48 and $72 \mathrm{~h}$ following transfection, and cell apoptosis analysis was performed $24 \mathrm{~h}$ following transfection. The untransfected cells were also used as blank controls.

Cell proliferation assay. Cellular growth was analyzed by the 3-(4,5-dimethylthiazol-2-yl)-2,5-diphenyltetrazolium bromide 

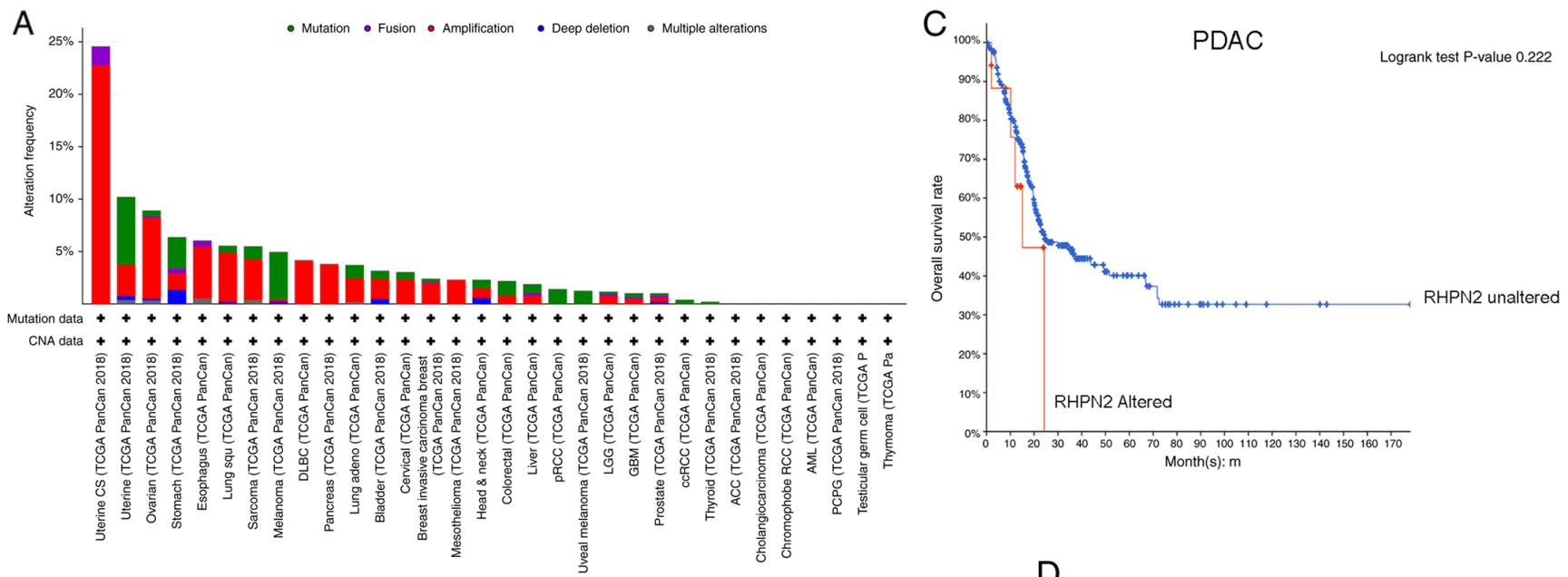

B

Pan-cancer analysis

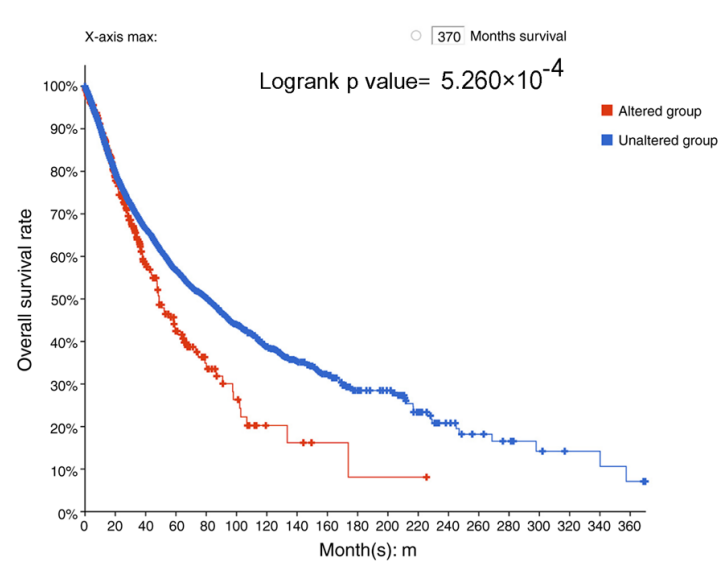

$\mathrm{D}$

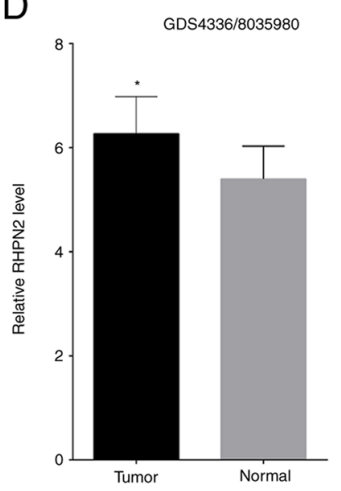

Figure 1. RHPN2 expression and patient survival associated with RHPN2 expression in pan-cancer and in PDAC. (A) Alteration of RHPN2 in pan-cancer. (B) Kaplan-Meir curves and log-rank test of the overall survival of pan-cancer patients according to RHPN2 alteration. (C) Kaplan-Meier curves and log-rank test of the overall survival of patients with PDAC according to RHPN2 alteration. (D) Gene Expression Omnibus (GEO) dataset (GDS4336/8035980) analysis for the RHPN2 expression in 45 pairs of tumor tissues and adjacent normal tissues from patients with PDAC, RHPN2 expression was compared using the paired t-test. "P<0.05. RHPN2, rhophilin 2; PDAC, pancreatic ductal adenocarcinoma; TCGA, The Cancer Genome Atlas.

(MTT)-based colorimetric assay (9-13). Briefly, cells were placed into 96 -well plates at a density of $5 \times 10^{5} /$ well. MTT reagent was added to the medium at a final concentration of $0.1 \mathrm{mg} / \mathrm{ml}$. After the formation of insoluble formazan, $100 \mu \mathrm{l}$ of dimethyl sulfoxide was added to each well to solubilize the formazan. The optical density was measured on a microplate reader equipped with a $570 \mathrm{~nm}$ filter.

Cell apoptosis analysis. Transfected PANC1 cells and SW1990 cells were suspended at $5 \times 10^{5} / \mathrm{ml}$ in Annexin V-fluorescein isothiocyanate (FITC) (Abcam) in Annexin binding buffer for flow cytometry (cat. no. V13246; Thermo Fisher Scientific Inc.). The suspension was incubated for $15 \mathrm{~min}$ at room temperature followed by the addition of $0.5 \mu l$ propidium iodide (PI; Abcam) to each sample. Samples were analyzed using a FACSCalibur instrument (BD Biosciences) using a $488 \mathrm{~nm}$ excitation line (argon ion laser or solid-state laser) and emission was detected at $530 \mathrm{~nm}$ (for FITC) and 575-610 nm (for PI). The data were analyzed using the BD FACSuite ${ }^{\mathrm{TM}}$ version 1.01 (BD Biosciences). Early stage apoptosis was assessed.

Statistical analyses. All the experiments were repeated three times. Data are presented as mean \pm standard devia- tion (SD). Survival data were analyzed using the log-rank test. Kaplan-Meier analysis was used for generation of the survival curves. Paired two-tailed Student's t-tests were used to analyze the mean values of paired groups (tumor and normal tissue from the same patient). Unpaired two-tailed Student's t-tests were used to analyze the mean values of the two PDAC cell lines. One-way ANOVA was used followed by the post hoc Tukey's test was used for multiple comparisons. All calculations were performed using SPSS software (version 16.0; SPSS Inc.). P $<0.05$ was considered to indicate a statistically significant difference.

\section{Results}

Bioinformatics analysis of RHPN2 in pan-cancer and patients with pancreatic cancer. Investigation of the frequency of alterations of RHPN2 in pan-cancer analysis revealed that the predominant alteration was amplification (Fig. 1A). Pan-cancer patients were divided into two groups, RHPN2-altered and RHPN2-unaltered, and the patients' survival according to their RHPN2 alteration status was tested. The results revealed that patients with pan-cancer in the RHPN2-altered group had a lower overall survival rate compared with the RHPN2-unaltered group (Fig. 1B). Next, the survival rate of patients with pancreatic cancer was tested 
A
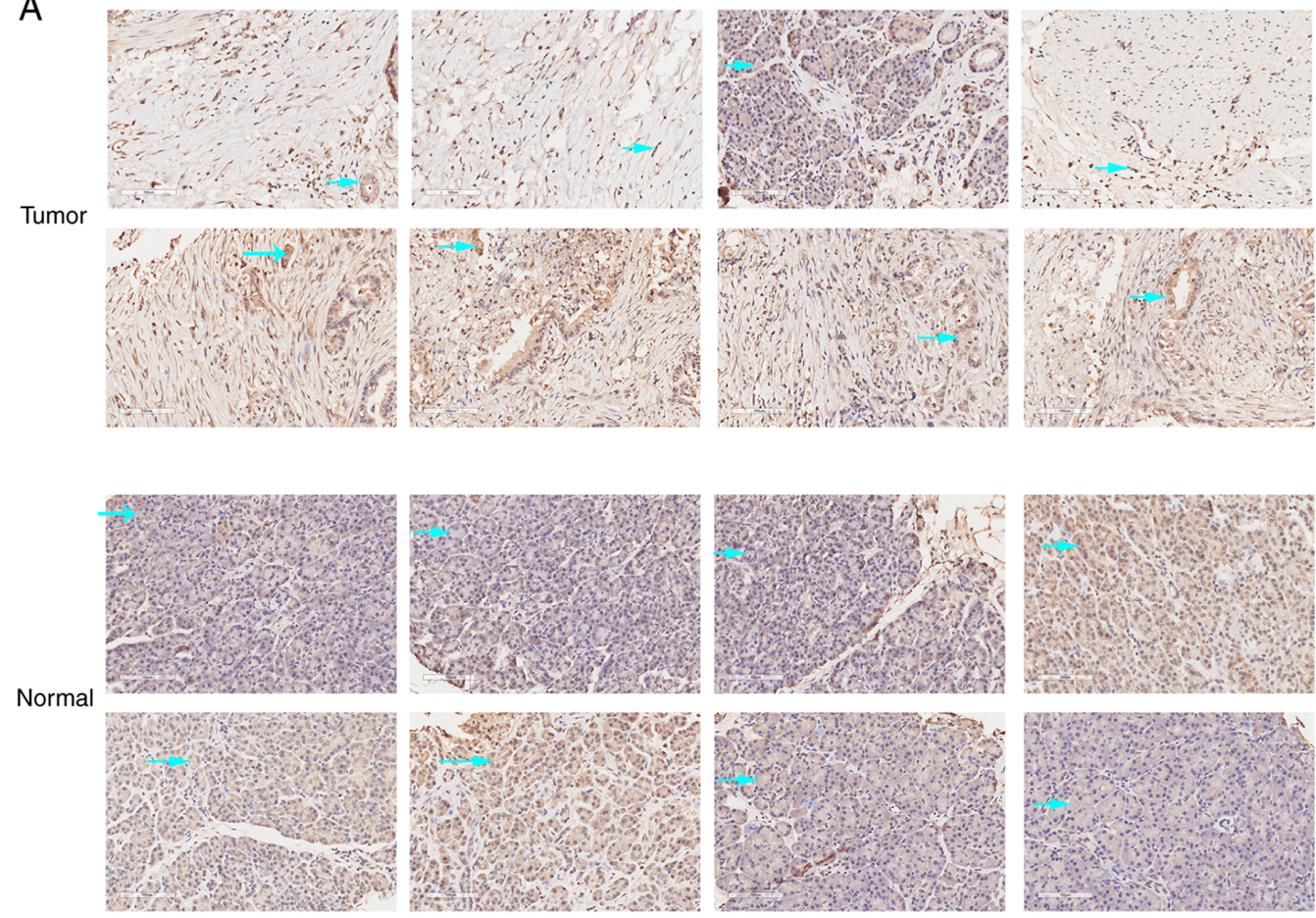

B
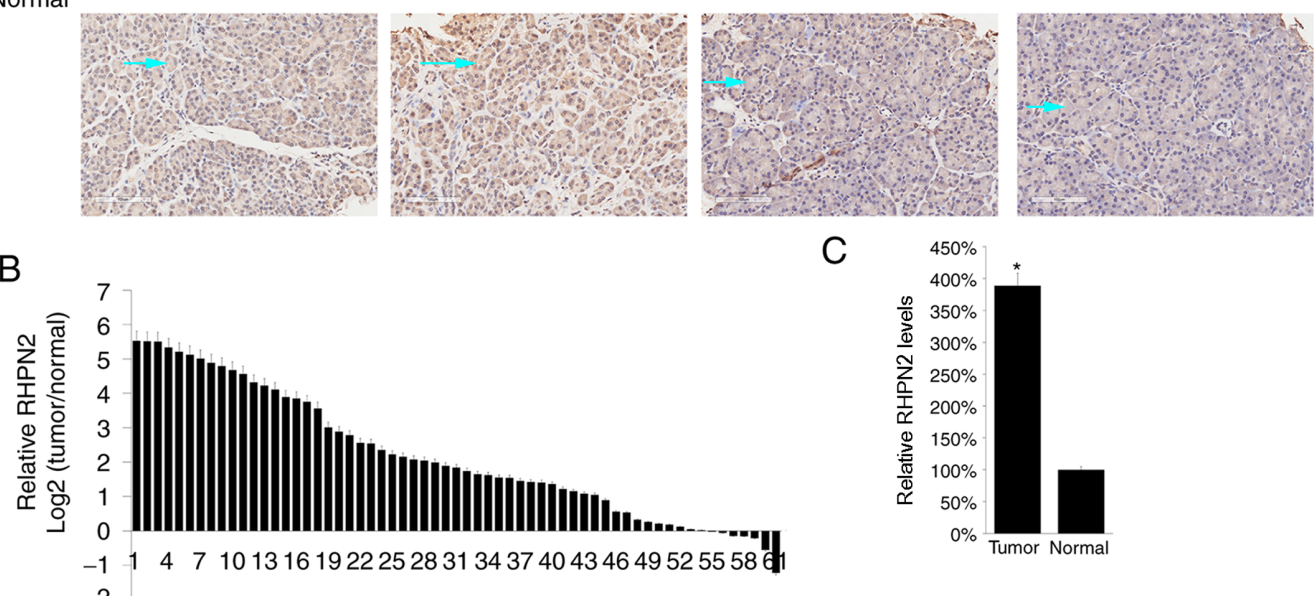

C

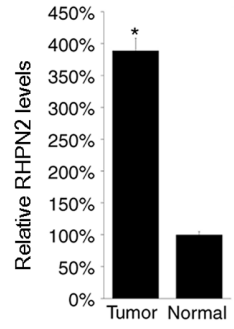

D Overall survival

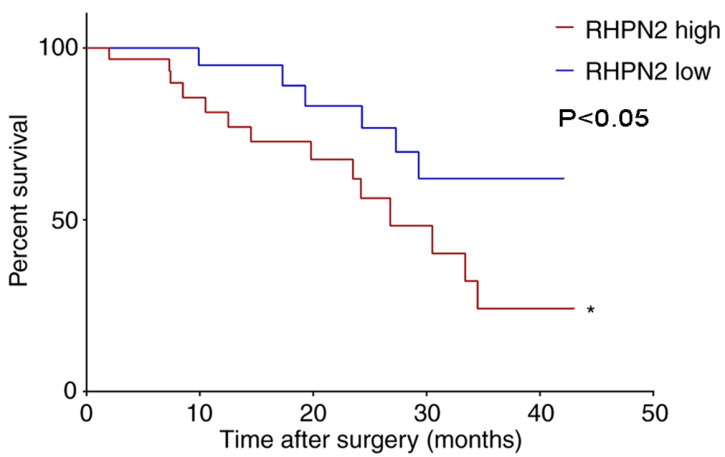

Figure 2. PDAC tissues have higher levels of RHPN2 mRNA and protein levels compared with normal tissues. (A) Representative immunohistochemistry images of normal and tumor samples from patients with PDAC stained with RHPN2 (magnification, x200). RHPN2 expression in the cytoplasm was denoted by the blue arrow. (B) Quantification of RHPN2 mRNA levels in the 61 tumor and paired normal adjacent tissues from PDAC patients. (C) Mean values of RHPN2 mRNA expression in the 61 tumor and paired normal adjacent tissues from PDAC patients were calculated. Data are presented as mean \pm SD (D) Survival curves of RHPN2-high vs. RHPN2-low patients with PDAC across 50 months, with analysis performed by the log-rank test. Each experiment was repeated at least three times. " $\mathrm{P}<0.05$. RHPN2, rhophilin 2; PDAC, pancreatic ductal adenocarcinoma.

according to whether RHPN2 was altered or not (Fig. 1C), and it was demonstrated that the difference between the RHPN2-altered and RHPN2-unaltered groups was not significant; which was may be due to the limited number of patients. Finally, the GEO dataset for RHPN2 expression in 45 pairs of tumor tissues and adjacent normal tissues (GDS4336/8035980) was assessed and it was demonstrated that tumor tissues showed higher RHPN2 levels compared with adjacent normal tissues (Fig. 1D).
High RHPN2 levels in PDAC tissues are correlated with low survival rate. To further study the role of RHPN2, PDAC tissues with matched adjacent normal tissues from 61 patients from the Sichuan Provincial Cancer Hospital (Chengdu, China) were collected. The clinical information of the 61 patients with PDAC are listed in Table SI. Majority of the patients had stage IIB PDAC (Table SI). RHPN2 protein levels were assessed by immunohistochemistry (IHC). Eight representative cases 

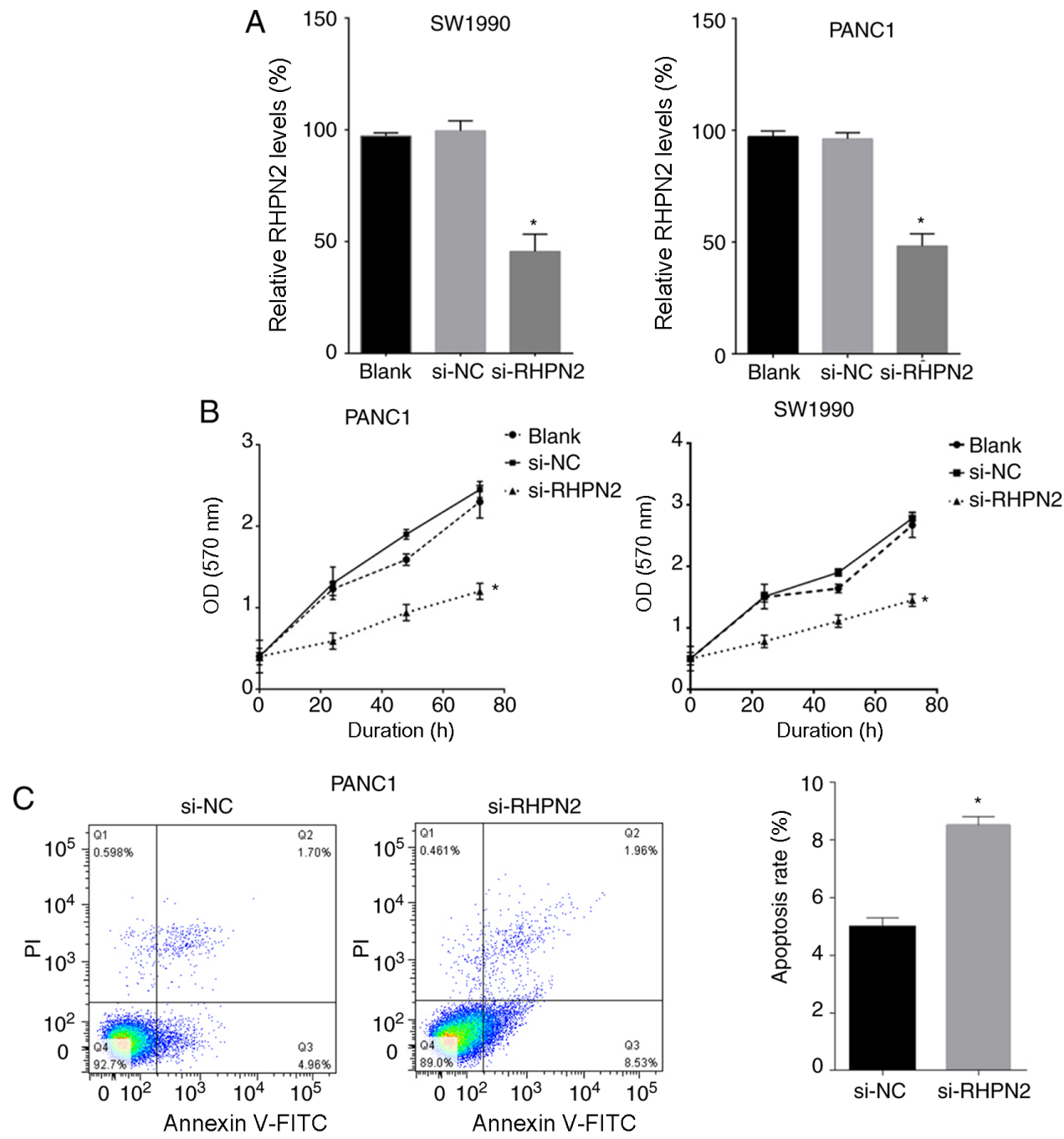

PANC1
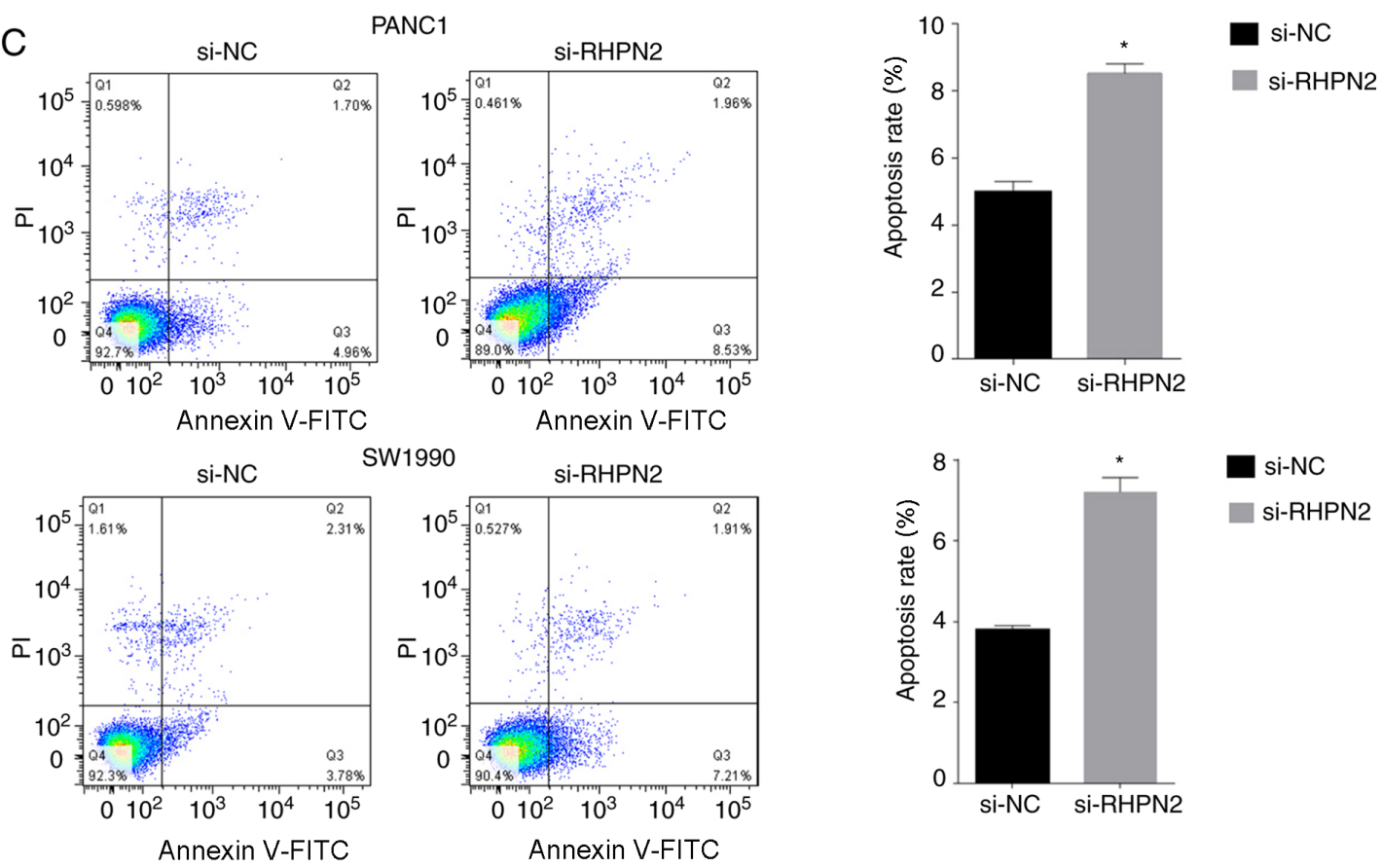

si-NC

si-RHPN2

Figure 3. Silencing of RHPN2 reduces PDAC cell proliferation and increases cell apoptosis rate. (A) PANC1 and SW1990 cells were transfected with si-RHPN2 and si-NC; $24 \mathrm{~h}$ later, RHPN2 mRNA was quantified by reverse-transcription quantitative PCR. RHPN2 mRNA levels of the si-NC transfection group were arbitrarily defined as $100 \%$. The blank group consisted of untransfected cells. (B) Cellular proliferation of PANC1 and SW1990 cells was tested by MTT analysis following si-RHPN2 and si-NC transfection. (C) Cell apoptosis rate tested by Annexin V-FITC/PI double staining $24 \mathrm{~h}$ after si-RHPN2 and si-NC transfection. Data are presented as the mean \pm SD. Each experiment was repeated at least three times. ${ }^{*} \mathrm{P}<0.05$. RHPN2, rhophilin 2; PDAC, pancreatic ductal adenocarcinoma; $\mathrm{NC}$, negative control; si-small interfering; PI, propidium iodide; OD, optical density.

were presented and it was demonstrated that RHPN2 is mostly expressed in the cytoplasm as denoted by the arrows (Fig. 2A). Next, RHPN2 mRNA levels were determined by RT-qPCR. The results demonstrated that RHPN2 mRNA levels were higher in tumor tissue compared with normal tissues (Fig. 2B), and the mean value of mRNA RHPN2 levels in tumor tissues was higher compared with that in normal tissues (Fig. 2C).In addition, the relationship between RHPN2 and the survival of patients with PDAC was assessed by dividing patients into
RHPN2-high vs. RHPN2-low groups according to the median value of RHPN2 (3.59). Patient survival was followed across 50 months and the RHPN2-high group had a lower survival rate compared with the RHPN2-low group (Fig. 2D).

RHPN2 inhibition reduces PDAC cell proliferation and increases apoptosis rate. PANC1 and SW1990 cell lines were used for testing the effects of RHPN2 on cultured PDAC cells. PANC1 and SW1990 cells were transfected with si-RHPN2. 

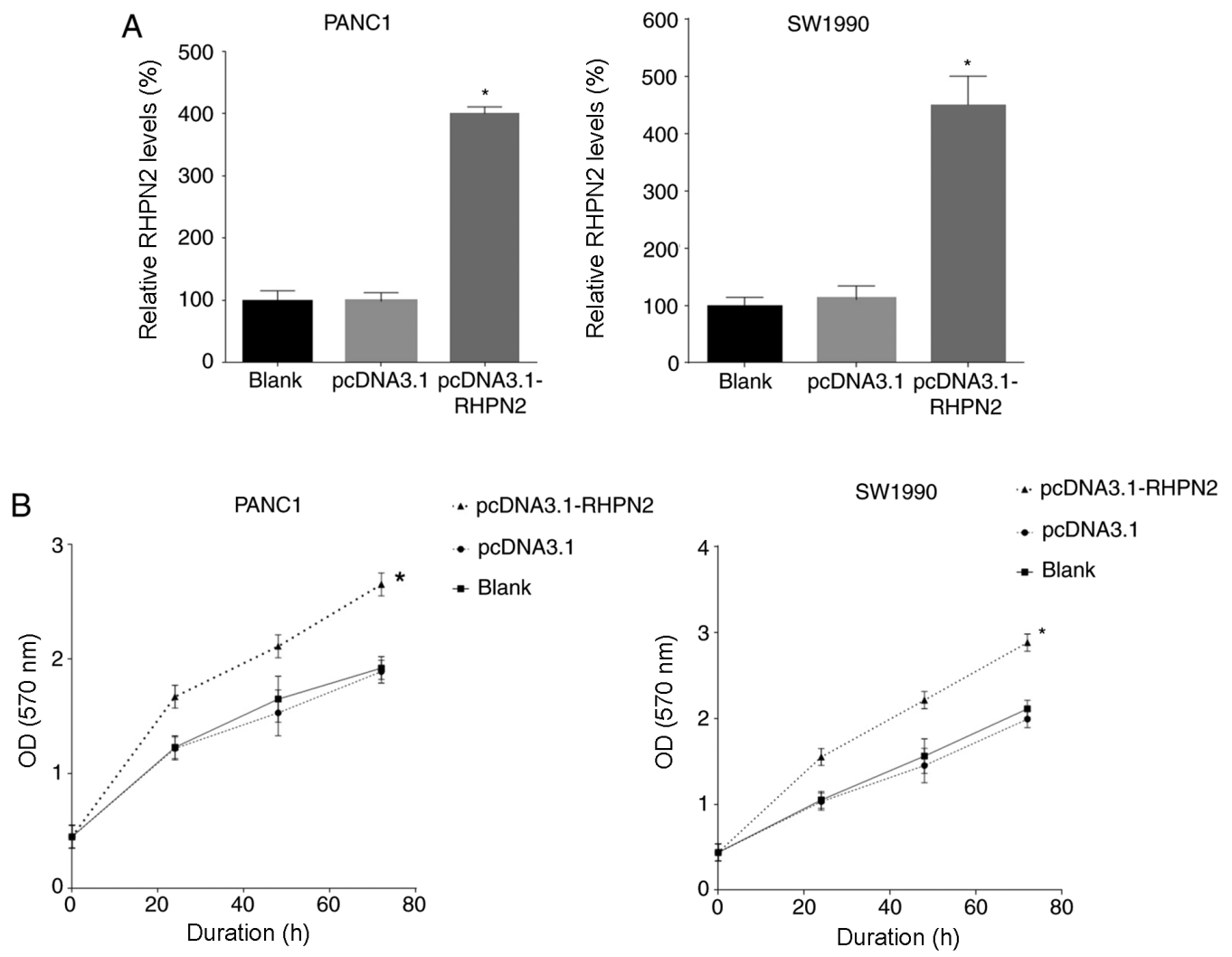

Figure 4. Overexpression of RHPN2 promotes PDAC cell proliferation. (A) PANC1 and SW1990 cells were transfected with pcDNA3.1-RHPN2 and pcDNA3.1 (negative control); $24 \mathrm{~h}$ later, RHPN2 mRNA levels were tested by reverse-transcription quantitative PCR. RHPN2 mRNA levels of the pcDNA3.1 transfection group were arbitrarily defined as 100\%. The untransfected cell were defined as the blank group. (B) The cellular proliferation of PANC1 and SW1990 cells tested by MTT analysis following pcDNA3.1-RHPN2 and pcDNA3.1 transfection. Data are presented as mean $\pm \mathrm{SD}$, and each experiment was repeated at least three times. "P<0.05. RHPN2, rhophilin 2; PDAC, pancreatic ductal adenocarcinoma; OD, optical density; pcDNA3.1, empty vector.

The downregulation of RHPN2 mRNA levels was confirmed by RT-qPCR in both cell lines (Fig. 3A). The proliferation of PANC1 and SW1990 cells was tested by the MTT assay, which demonstrated that inhibition of RHPN2 reduced PDAC cell proliferation (Fig. 3B). Cell apoptosis was assayed using Annexin V/propidium iodide (PI) double-staining. Downregulation of RHPN2 increased the apoptosis rate of PANC1 and SW1990 cells compared with the negative control (Fig. 3C).

Overexpression of RHPN2 promotes PDAC cell proliferation. Next, RHPN2 was overexpressed in PANC1 and SW1990 cells by transfecting with RHPN2 overexpression plasmid (pcDNA3.1-RHPN2). RHPN2 levels were tested $12 \mathrm{~h}$ later by RT-qPCR and the results demonstrated that RHPN2 expression was upregulated by plasmid transfection (Fig. 4A). The proliferation of PANC1 and SW1990 cells was tested by the MTT assay and the results demonstrated that overexpression of RHPN2 promoted PANC1 and SW1990 cell proliferation (Fig. 4B).

CEP78 expression is negatively associated with RHPN2 expression. A previous study demonstrated that RHPN2 activated RhoA (10). In the present study, the network of RHPN2 was constructed and it was revealed that CEP78 was one of the proteins that interacted with RHPN2. The alteration frequency of CEP78 in pan-cancer analysis was assessed and the findings revealed that the most frequent alteration of CEP78 was mutation (Fig. 5B). Subsequently, the CEP78 mRNA levels were analyzed by RT-qPCR, and relative CEP78 levels [ $\log _{2}$ Tumor/Normal)] were calculated. The present study demonstrated that CEP78 mRNA levels were lower in tumor tissues (Fig. 5C), and the mean value of CEP78 levels in tumor tissues was lower compared with CEP78 levels in normal tissues (Fig. 5D). In addition, the significance of CEP78 in patients with PDAC survival was assessed after dividing the 61 patients into CEP78-high vs. CEP78-low groups according to the median value of CEP78 (2.78). Patient survival was followed across 50 months, and we found that the CEP78-low group had a lower survival rate compared with the CEP-78 high group (Fig. 5E), which is consistent with a previous study that demonstrated that low expression of CEP78 is associated with poor prognosis of patients with colorectal cancer (20). Next, to evaluate whether the expression of RHPN2 is related with that of CEP78, RHPN2 levels were overexpressed or silenced in cultured PDAC cells by transfection of pcDNA3.1-RHPN2 and si-RHPN2, respectively and it was demonstrated that overexpression of RHPN2 decreased CEP78 levels (Fig. 5F) and silencing of RHPN2 increased CEP78 levels (Fig. 5G) in both PANC1 and SW1990 cells. The aforementioned results indicate that CEP78 is involved in RNPN2 function.

RHPN2 is the target gene of miR-144-3p, miR-141-3p, $m i R-96-5 p, m i R-200 a$ and miR-183-5p. Bioinformatics analysis in the present study indicated RHPN2 is the target 
A

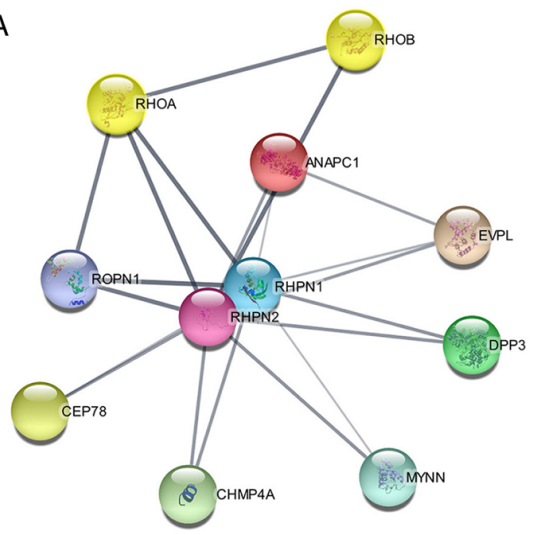

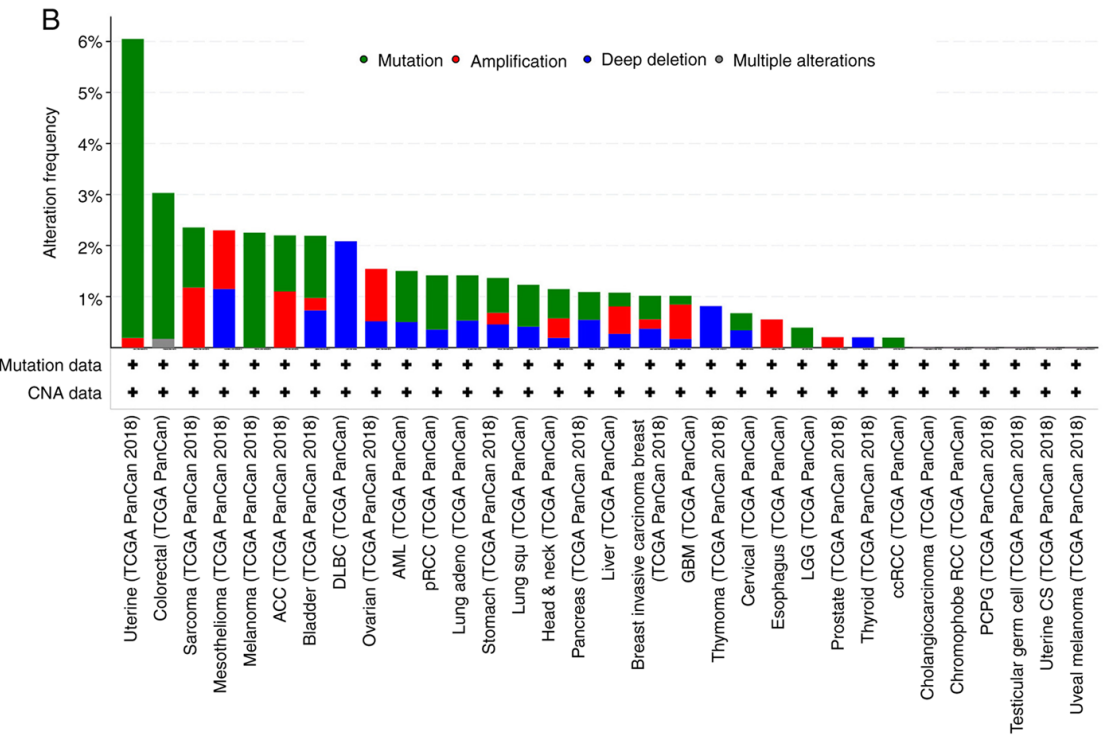

E

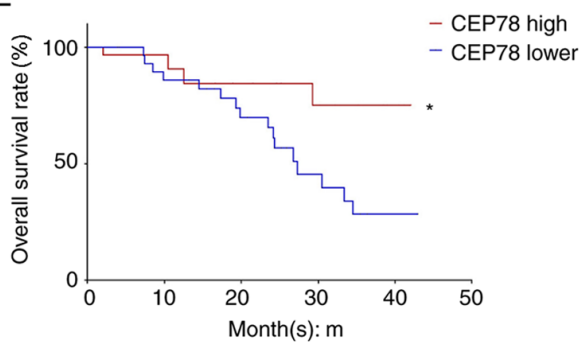

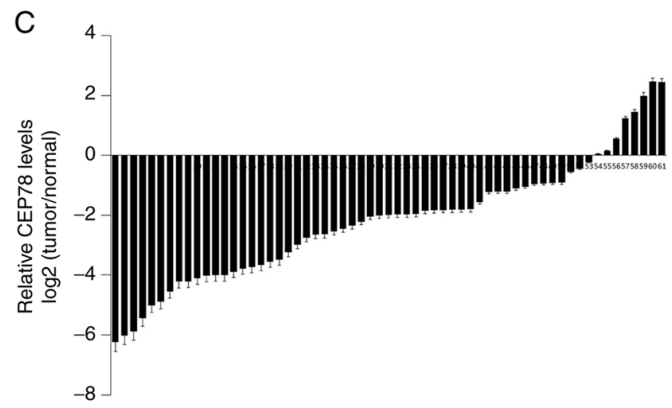
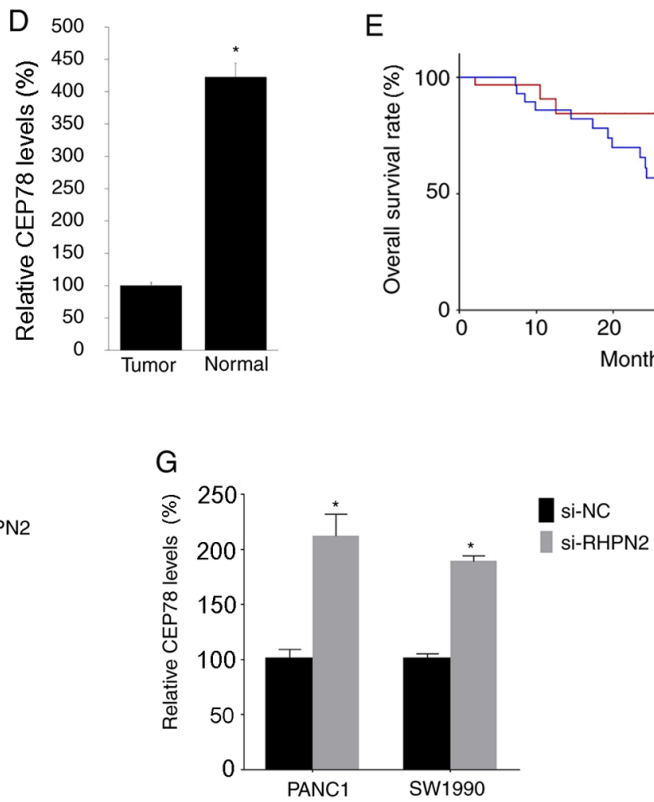

Figure 5. RHPN2 network and correlation analysis. (A) The RHPN2 network as revealed by Cytoscape software. (B) The amplification of CEP78 across various types of cancer. (C) CEP78 mRNAs levels in the 61 tumor samples and matching normal adjacent tissues were quantified by RT-qPCR. (D) The mean value of CEP78 mRNA expression in tumor tissue and normal adjacent tissues. (E) CEP78-high vs. CEP78-low groups and patient survival across 50 months, with survival analysis performed by log-rank test. (F) PANC1 and SW1990 cells were transfected with pcDNA3.1-RHPN2 separately; $24 \mathrm{~h}$ later, CEP78 levels were tested by RT-qPCR. (G) PANC1 and SW1990 cells were transfected with si-RHPN2 separately; $24 \mathrm{~h}$ later, the CEP78 levels were tested by RT-qPCR. Data are presented as mean $\pm \mathrm{SD}$, and each experiment was repeated at least three times. "P $<0.05$. RHPN2, rhophilin 2; PDAC, pancreatic ductal adenocarcinoma; pcDNA3.1, empty vector; CEP78, centrosomal protein 78; NC, negative control; si, small interfering; RT-q, reverse-transcription quantitative; TCGA, The Cancer Genome Atlas.

of some important microRNAs (miR) including miR-144-3p, miR-141-3p, miR-96-5p, miR-200a and miR-183-5p (Fig. S1).

\section{Discussion}

In the present study, the function of RHPN2 in PDAC was assessed and the findings revealed that the level of RHPN2 was higher in PDAC tumor tissues compared with adjacent normal tissue. Notably, high RHPN2 levels in PDAC tissues were associated with a low survival rate in patients with PDAC. As the overall 5-year survival rate of PDAC is $7.1 \%$ (21), identification of a novel gene related to the survival of PDAC patients is critically important. In addition, in the present study RHPN2 overexpres- sion promoted the growth of PDAC cells and RHPN2 inhibition promoted cell apoptosis. Hence, PDAC growth promoted by RHPN2 may increase the death rate among patients with PDAC.

To the best of our knowledge, this is the first report of the function of RHPN2 in PDAC. Until now, the oncogenic function of RHPN2 has been studied only in malignant glioma; the aforementioned study demonstrated that RHPN2 drives mesenchymal transformation by triggering RhoA activation (10).

Notably, the data from network analysis in the present study demonstrated that both RHPN1 and CEP78 have a close relationship with RHPN2. The role of RHPN1 in cancer is still unknown (22); and it is hypothesized that RHPN1 may play a 
role similar to RHPN2. The present study demonstrated that the levels of CEP78 are low in PDAC tumor tissues compared with normal tissue, and that higher levels of CEP78 in tumor tissue were associated with increased survival rate in patients with PDAC. The molecular interaction between RHPN2 and CEP78 remains unknown; it is possible that RHPN2 may inhibit CEP78, which could be reintroduced in PDAC cells resulting in $\mathrm{G} 2 / \mathrm{M}$ phase arrest. Future studies can investigate the aforementioned phenomenon.

Bioinformatics analysis in the present study indicated RHPN2 is the target of some important microRNAs (miR) including miR-144-3p, miR-141-3p, miR-96-5p, miR-200a and miR-183-5p. These microRNAs serve important roles in the molecular mechanisms of some types of cancer. For example, miR-144-3p acts as a suppressive factor in laryngeal squamous cell carcinoma (23), gastric cancer (24), hepatocellular carcinoma (25), and pancreatic cancer (26). miR-141-3p inhibits colorectal cancer, its overexpression significantly delayed colorectal cancer progression (27). miR-96-5p and miR-200a also have a suppressive role in certain types of cancer, such as breast cancer, ovarian cancer, oral carcinoma and gastric adenocarcinoma (28-34). On the other hand, miR-183-5p promotes cell proliferation, migration, and cell cycling in non-small cell lung cancer (35).

A limitation of the present study was that the relationship between microRNAs and RHPN2 and the downstream molecular of RHPN2 was not investigated. Future studies can investigate this.

In conclusion, the findings of the present study demonstrated that RHPN2 may promote PDAC and is therefore a promising candidate for targeted therapy, which is vitally needed for patients with PDAC whose prospects for survival are dismal.

\section{Acknowledgements}

The authors would like to thank Dr Bo Yuan (Department of General Practice, West China Hospital, Sichuan University) for discussions of the present study.

\section{Funding}

No funding was received.

\section{Availability of data and materials}

The datasets used and/or analyzed during the current study are available from the corresponding author on reasonable request.

\section{Authors' contributions}

WB collected the patient data and performed the bioinformatics analysis. XF performed PCR and transfection. XT and WB performed the apoptosis analysis. XT contributed to the study design and manuscript writing. All authors read and approved the final manuscript.

\section{Ethics approval and consent to participate}

The present study was approved by the Ethics Committee of Sichuan University (Chengdu, China; approval no. 20191109) and written informed consent was provided by all patients enrolled.

\section{Patient consent for publication}

Not applicable.

\section{Competing interests}

The authors declare that they have no competing interests.

\section{References}

1. Bray F, Ferlay J, Soerjomataram I, Siegel RL, Torre LA and Jemal A: Global cancer statistics 2018: GLOBOCAN estimates of incidence and mortality worldwide for 36 cancers in 185 countries. CA Cancer J Clin 68: 394-424, 2018.

2. Ferlay J, Ervik M,Lam F, Colombet M, Mery L, Piñeros M, Znaor A, Soerjomataram I and Bray F: Global Cancer Observatory: Cancer Today. International Agency for Research on Cancer, Lyon, 2018.

3. Yang J, Siri JG, Remais JV, Cheng Q, Zhang H, Chan KK, Sun Z, Zhao Y, Cong N, Li X, et al: The Tsinghua-Lancet Commission on Healthy Cities in China: Unlocking the power of cities for a healthy China. Lancet 391: 2140-2184, 2018.

4. Yadav D and Lowenfels AB: The Epidemiology of Pancreatitis and Pancreatic Cancer. Gastroenterology 144: 1252-1261, 2013.

5. Chen W, Zheng R, Zhang S, Zhao P, Zeng H and Zou X: Report of cancer incidence and mortality in China, 2010. Ann Transl Med 2: 61, 2014.

6. Hidalgo M, Cascinu S, Kleeff J, Labianca R, Löhr JM, Neoptolemos J, Real FX, Van Laethem JL and Heinemann V: Addressing the challenges of pancreatic cancer: Future directions for improving outcomes. Pancreatology 15: 8-18, 2015.

7. Lambert A, Schwarz L, Borbath I, Henry A, Van Laethem JL, MalkaD,Ducreux Mand Conroy T: Anupdate on treatmentoptions for pancreatic adenocarcinoma. Ther Adv Med Oncol: Sep 25, 2019 (Epub ahead of print). doi: 10.1177/1758835919875568.

8. Hammel P, Huguet F, van Laethem JL, Goldstein D, Glimelius B, Artru P, Borbath I, Bouché O, Shannon J, André T, et al; LAP07 Trial Group: effect of chemoradiotherapy vs. chemotherapy on survival in patients with locally advanced pancreatic cancer controlled after 4 months of gemcitabine with or without erlotinib: The LAP07 Randomized Clinical Trial. JAMA 315: 1844-1853, 2016.

9. O'Reilly EM and Hechtman JF: Tumour response to TRK inhibition in a patient with pancreatic adenocarcinoma harbouring an NTRK gene fusion. Ann Oncol 30: viii36-viii40, 2019.

10. Danussi C, Akavia UD, Niola F, Jovic A, Lasorella A, Pe'er D and Iavarone A: RHPN2 drives mesenchymal transformation in malignant glioma by triggering RhoA activation. Cancer Res 73: 5140-5150, 2013.

11. Chen Y, Sheng R, Källberg M, Silkov A, Tun MP, Bhardwaj N, Kurilova S, Hall RA, Honig B, Lu H, et al: Genome-wide functional annotation of dual-specificity protein- and lipid-binding modules that regulate protein interactions. Mol Cell 46: 226-237, 2012.

12. Steuve S, Devosse T, Lauwers E, Vanderwinden JM, André B, Courtoy PJ and Pirson I: Rhophilin-2 is targeted to late-endosomal structures of the vesicular machinery in the presence of activated RhoB. Exp Cell Res 312: 3981-3989, 2006.

13. Cerami E, Gao J, Dogrusoz U, Gross BE, Sumer SO, Aksoy BA, Jacobsen A, Byrne CJ, Heuer ML, Larsson E, et al: The cBio cancer genomics portal: An open platform for exploring multidimensional cancer genomics data. Cancer Discov 2: 401-404, 2012.

14. Gao J, Aksoy BA, Dogrusoz U, Dresdner G, Gross B, Sumer SO, Sun Y, Jacobsen A, Sinha R, Larsson E, et al: Integrative analysis of complex cancer genomics and clinical profiles using the cBioPortal. Sci Signal 6: pl1, 2013.

15. Zhang G, He P, Tan H, Budhu A, Gaedcke J, Ghadimi BM, Ried T, Yfantis HG, Lee DH, Maitra A, et al: Integration of metabolomics and transcriptomics revealed a fatty acid network exerting growth inhibitory effects in human pancreatic cancer. Clin Cancer Res 19: 4983-4993, 2013.

16. Kohl M, Wiese S and Warscheid B: Cytoscape: software for visualization and analysis of biological networks. In: Data Mining in Proteomics. Springer, pp291-303, 2011. 
17. Amin MB, Edge S, Greene F, Byrd DR, Brookland RK, Washington MK, Gershenwald JE, Compton CC, Hess KR, et al. (eds): AJCC Cancer Staging Manual. 8th edition. Springer, 2017.

18. Fahimi HD: Cytochemical detection of peroxisomes in light and electron microscopy with 3,3'-diaminobenzidine. Methods Mol Biol 1595: 93-100, 2017.

19. Livak KJ and Schmittgen TD: Analysis of relative gene expression data using real-time quantitative PCR and the 2(-Delta Delta C(T)) method. Methods 25: 402-408, 2001

20. Zhang M, Duan T, Wang L, Tang J, Luo R, Zhang R and Kang T: Low expression of centrosomal protein 78 (CEP78) is associated with poor prognosis of colorectal cancer patients. Chin J Cancer 35: 62, 2016.

21. Stark AP, Sacks GD, Rochefort MM, Donahue TR, Reber HA, Tomlinson JS, Dawson DW, Eibl G and Hines OJ: Long-term survival in patients with pancreatic ductal adenocarcinoma. Surgery 159: 1520-1527, 2016.

22. Lal MA, Andersson AC, Katayama K, Xiao Z, Nukui M, Hultenby K, Wernerson A and Tryggvason K: Rhophilin-1 is a key regulator of the podocyte cytoskeleton and is essential for glomerular filtration. J Am Soc Nephrol 26: 647-662, 2015.

23. Zhang SY, Lu ZM, Lin YF, Chen LS, Luo XN, Song XH, Chen SH and Wu YL: miR-144-3p, a tumor suppressive microRNA targeting ETS-1 in laryngeal squamous cell carcinoma. Oncotarget 7: 11637-11650, 2016

24. Li B, Zhang S, Shen H and Li C: MicroRNA-144-3p suppresses gastric cancer progression by inhibiting epithelial-to-mesenchymal transition through targeting PBX3. Biochem Biophys Res Commun 484: 241-247, 2017.

25. Liang HW, Ye ZH, Yin SY, Mo WJ, Wang HL, Zhao JC, Liang GM, Feng ZB, Chen G and Luo DZ: A comprehensive insight into the clinicopathologic significance of miR-144-3p in hepatocellular carcinoma. OncoTargets Ther 10: 3405-3419, 2017.

26. Li J, Sun P, Yue Z, Zhang D, You K and Wang J: miR-144-3p induces cell cycle arrest and apoptosis in pancreatic cancer cells by targeting proline-rich protein 11 expression via the mitogen-activated protein kinase signaling pathway. DNA Cell Biol 36: 619-626, 2017.
27. Liang Z, Li X, Liu S, Li C, Wang X and Xing J: MiR-141-3p inhibits cell proliferation, migration and invasion by targeting TRAF5 in colorectal cancer. Biochem Biophys Res Commun 514: 699-705, 2019.

28. Shi Y, Zhao Y, Shao N, Ye R, Lin Y, Zhang N, Li W, Zhang Y and Wang S: Overexpression of microRNA-96-5p inhibits autophagy and apoptosis and enhances the proliferation, migration and invasiveness of human breast cancer cells. Oncol Lett 13: 4402-4412, 2017.

29. Liu B, Zhang J and Yang D: miR-96-5p promotes the proliferation and migration of ovarian cancer cells by suppressing Caveolael. J Ovarian Res 12: 57, 2019.

30. Wang H, Ma N, Li W and Wang Z: MicroRNA-96-5p promotes proliferation, invasion and EMT of oral carcinoma cells by directly targeting FOXF2. Biol Open 9: 9, 2020.

31. Zhou HY, Wu CQ and Bi EX: MiR-96-5p inhibition induces cell apoptosis in gastric adenocarcinoma. World J Gastroenterol 25: 6823-6834, 2019

32. Shi C, Yang Y, Zhang L, Yu J, Qin S, Xu H and Gao Y: MiR-200a-3p promoted the malignant behaviors of ovarian cancer cells through regulating PCDH9. OncoTargets Ther 12: 8329-8338, 2019.

33. Tang W, Yu X, Zeng R and Chen L: LncRNA-ATB promotes cisplatin resistance in lung adenocarcinoma cells by targeting the miR-200a/ $\beta$-catenin pathway. Cancer Manag Res 12: 2001-2014, 2020.

34. Liu J, Ke F, Chen T, Zhou Q, Weng L, Tan J, Shen W, Li L, Zhou J, Xu C, et al: MicroRNAs that regulate PTEN as potential biomarkers in colorectal cancer: A systematic review. J Cancer Res Clin Oncol 146: 809-820, 2020.

35. Wang H, Ma Z, Liu X, Zhang C, Hu Y, Ding L, Qi P, Wang J, Lu S and Li Y: MiR-183-5p is required for non-small cell lung cancer progression by repressing PTEN. Biomed Pharmacother 111: 1103-1111, 2019.

This work is licensed under a Creative Commons Attribution-NonCommercial-NoDerivatives 4.0 International (CC BY-NC-ND 4.0) License. 